\title{
A Single Center Study Comparing the Stainable Iron Depositions in 1000 Explanted Cirrhotic Livers of Different Causes
}

\author{
Bita Geramizadeh, ${ }^{1,2,}$ Yalda Ghazanfari, ${ }^{2}$ Saman Nikeghbalian, ${ }^{3}$ and Seyed-AliMalekhosseini ${ }^{3}$ \\ ${ }^{1}$ Department of Pathology, Transplant Research Center, Shiraz University of Medical Sciences, Shiraz, IR Iran \\ ${ }^{2}$ Department of Pathology, Shiraz University of Medical Sciences, Shiraz, IR Iran \\ 3 Department of Surgery, Shiraz University of Medical Sciences, Shiraz, IR Iran \\ ${ }^{*}$ Corresponding Author: Bita Geramizadeh, Department of Pathology, Transplant Research Center, Shiraz University of Medical Sciences, Shiraz, IR Iran. Tel/Fax: +98-7136473238, \\ E-mail:geramib@gmail.com
}

Received 2015 October 9; Revised 2015 November 23; Accepted 2015 November 28.

\begin{abstract}
Background: There have been very few studies evaluating the close association between excess iron and cirrhosis; however, cirrhosis could be regarded as an iron-loading disorder.

Objectives: In this study, the goal was to show the levels of the iron content in the liver tissue in certain types of cirrhosis.

Patients and Methods: In this 7 year study (2008-2014), in 1000 explanted livers, the amount of iron was scored and compared according to the cause of the cirrhosis. The amount of iron in the liver was determined via the histochemical staining of the liver tissue, using Prussian-blue staining. Additionally, in each patient, the serum iron was determined and compared according to the cause of cirrhosis. Results: The highest content of iron has been found in cirrhosis caused by chronic hepatitis (i.e. hepatitis B, C, and autoimmune hepatitis), as well as in alcoholic cirrhosis. The least amount of stainable iron has been shown in biliary cirrhosis.

Conclusions: The presence of high stainable iron in patients with cirrhosis, secondary to chronic viral hepatitis, autoimmune hepatitis, and alcoholic hepatitis, should not be considered indicative of the presence of hereditary hemochromatosis; however, in those patients with biliary cirrhosis, a high iron content is rare, and can be a sign of the presence of the high iron Fe(HFE) gene mutation, or another type of hereditary hemochromatosis.
\end{abstract}

Keywords: Liver, Iron, Cirrhosis

\section{Background}

Hepatic iron deposition unrelated to hereditary hemochromatosis is common in cirrhosis (1); however, the pathogenesis of iron overload in cirrhosis in the absence of hemochromatosis gene (high iron Fe, HFE) mutations is poorly understood (2). The increased deposition of iron within the liver causes the production of reactive oxygen species, which may lead to cell injury and cell death(1). Iron overload has been shown to impair the immune response of the liver. Moreover, there is significant controversy regarding the risk of developing hepatocellular carcinoma (HCC) in siderotic nodules in the livers of patients with cirrhosis, compared to cirrhotic patients with no liver siderosis (3). It seems that hepatic iron overload may have a detrimental effect on cirrhosis in certain cases (4). Overall, hepatic iron overload can be seen in various types of cirrhosis, such as hepatitis $\mathrm{C}$, or alcoholic and non-alcoholic fatty liver disease (5).

\section{Objectives}

In this report, the goal was to describe the stainable iron deposition in 1000 cirrhotic livers of different causes, to show the severity of iron deposition in the absence of hemochromatosis in cirrhotic livers, and to determine the differences in the hepatic tissue iron content in various cases of cirrhosis.

\section{Patients and Methods}

Over a period of 7 years (2008 - 2014), Prussian blue staining for iron in a representative section of 1000 explanted livers was reviewed, and the severity of iron deposition was graded according to the semi-quantitative scoring method by Deugnier et al.(5) (Figures 1-3). A total score of 36 meant that all of the components of the liver were loaded with iron, and a score of 0 meant that there was no iron in the representative section of the liver.

It is worth mentioning that in our center, for all of the explanted livers, a random section from the left lobe was routinely stained by the Prussian-blue staining method. Then, the Prussian-blue stain of each explanted liver was blindly reviewed and graded by a liver pathologist (BG). At the same time, haematoxylin and eosin ( $\mathrm{H}$ and

Copyright ( ) 2015, Kowsar Corp. This is an open-access article distributed under the terms of the Creative Commons Attribution-NonCommercial 4.0 International License (http://creativecommons.org/licenses/by-nc/4.0/) which permits copy and redistribute the material just in noncommercial usages, provided the original work is properly cited. 
E) stained slides were also reviewed, and the pathological diagnosis of the cause of the cirrhosis were matched with the clinical, imaging, and laboratory diagnoses, according to the patients' charts. The severity of the iron deposition was compared with the cause of the cirrhosis.

The explanted livers with causes other than cirrhosis, such as Budd-chiari syndrome, malignancies, and fulminant hepatitis, were excluded from this study. In addition, since our goal was to study the iron content of liver cirrhosis in the absence of hemochromatosis, those patients with cirrhosis caused by primary and secondary hemochromatosis (including hemoglobinopathies such as thalassemia) were also omitted from the study. Finally, those patients with cryptogenic cirrhosis were excluded as well.

All of the patients were analyzed for the HFE gene mutation, and there were no homozygote cases in the cirrhotic patients; however, about $28 \%$ of them were heterozygotes for H63D. Moreover, no C282Y mutations were found. Therefore, all the explanted livers in the study period, with the exception of the above etiologies (which have been omitted from the study), were included in the study.

Finally, the serum iron levels in these 1000 cases were extracted from their clinical charts.

\section{Results}

Table 1 shows the causes of cirrhosis in the 1000 explanted livers in this study, with the most common cause of cirrhosis (448 cases) being chronic hepatitis (caused by chronic hepatitis B and C, autoimmune hepatitis, and alcoholic hepatitis).

The second most common cause (194 cases) of cirrhosis was biliary, composed of primary sclerosing cholangitis (PSC), biliary atresia (BA), progressive familial intrahepatic cholestasis (PFIC), and primary biliary cirrhosis (PBC). The third most common cause was metabolic disease (180 cases), such as Wilson's disease, Crigler-Najjar syndrome, hypercholesterolemia, hyperoxaluria, tyrosinemia, and neonatal hepatitis. The least common cause of cirrhosis was nonalcoholic steatohepatitis (NASH), in 178 cases.

Table 1 shows the mean score of the iron content according to the cause of cirrhosis and, as the table shows, the highest liver content was in cirrhosis caused by chronic hepatitis (viral, autoimmune, and alcoholic). The second most common cause was NASH. However, the level of iron in the cases with chronic hepatitis was significantly higher than all of the other causes of cirrhosis (P value $<0.05$ ). The iron content in the cirrhotic livers caused by chronic hepatitis and NASH can be as high as 36; that is, all of the components of the liver (hepatocytes, sinusoids, biliary duct epithelial cells, etc.) are loaded with iron.

The last column of Table 1 shows the range of the iron score in each of the causes of cirrhosis. Overall, the serum iron level in these 1000 cases was between 45 and $220 \mu \mathrm{g} / \mathrm{dL}$ (normal range: 60 - $170 \mu \mathrm{g} / \mathrm{dL}$ ). Moreover, there were no statistically significant differences between the serum iron concentrations in the different causes of cirrhosis in these 1000 cases.

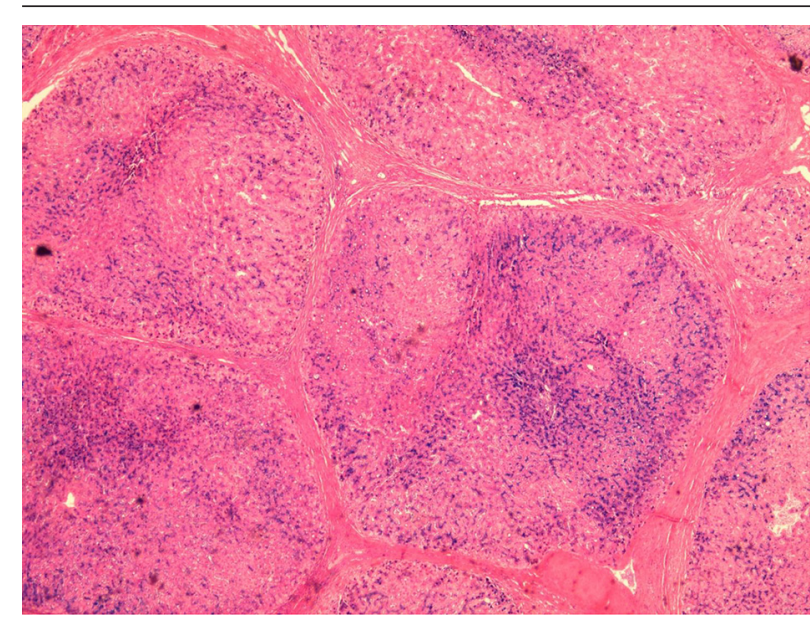

Figure 1. Low Power View of Iron Stain in a Case of Cirrhosis Caused by Chronic Hepatitis B, Showing Heavy Iron Deposition in the Cirrhotic Nodules $(\times 100)$

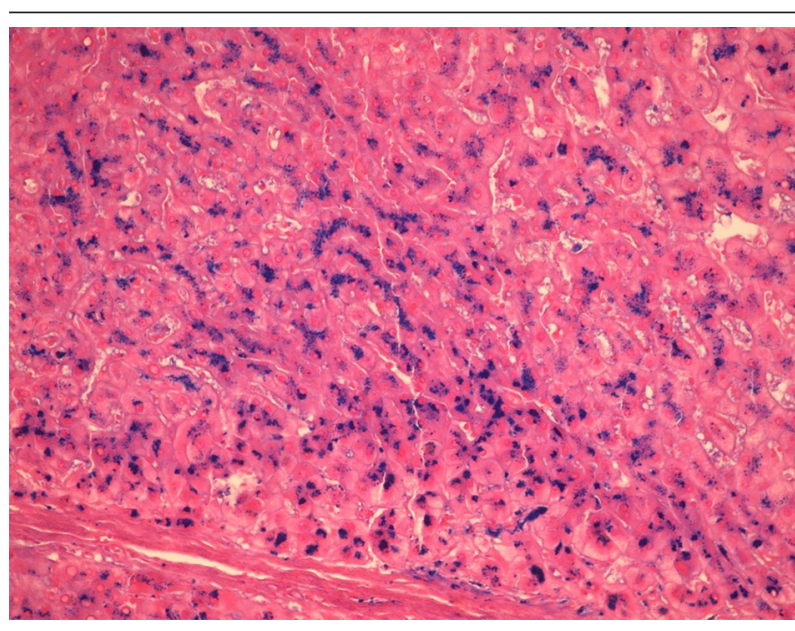

Figure 2. High Power View of Iron Staining in a Case of Cirrhosis Caused by Chronic Hepatitis C, Showing Heavy Iron Deposition in the Hepatocytes, Mostly in the Pericanalicular Area $(\times 400)$

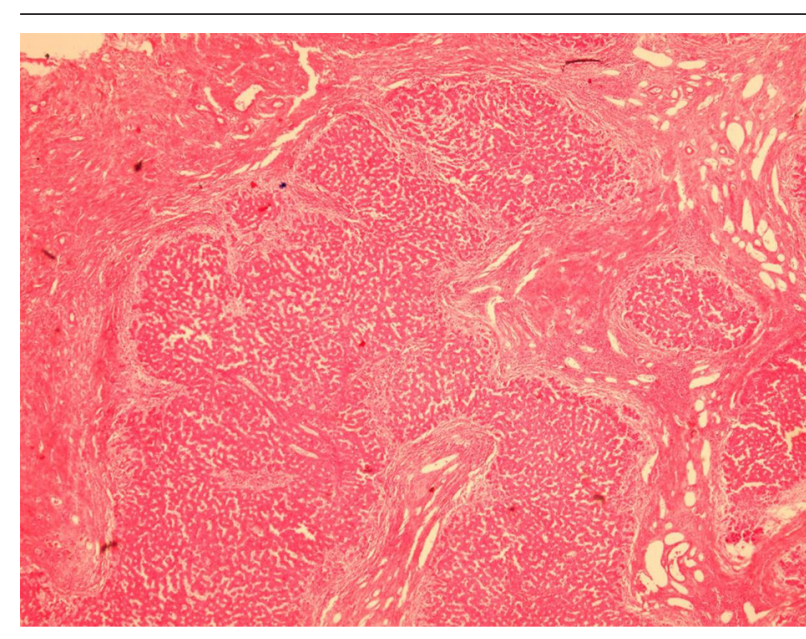

Figure 3. A Case of Biliary Type Cirrhosis, With no Stainable Iron $(\times 250)$ 
Geramizadeh B et al.

Table 1. The Causes of Cirrhosis in 1000 Explanted Livers, With Iron Scores and Serum Iron Levels

\begin{tabular}{lcccc}
\hline $\begin{array}{l}\text { Causes of } \\
\text { Cirrhosis }\end{array}$ & No. $(\%)$ & $\begin{array}{c}\text { Iron } \\
\text { Score }\end{array}$ & Scores & $\begin{array}{c}\text { Serum } \\
\text { Iron, } \mu \text { g/dL }\end{array}$ \\
\hline Chronic hepatitis & $448(44.8)$ & $8.48 \pm 4.23$ & $0-36$ & $150 \pm 56$ \\
$\begin{array}{l}\text { Nonalcoholic } \\
\text { steatohepatitis }\end{array}$ & $178(17.8)$ & $7.03 \pm 2.72$ & $0-36$ & $146 \pm 45$ \\
Metabolic & $180(18)$ & $4.47 \pm 1.32$ & $0-27$ & $110 \pm 35$ \\
Biliary & $194(19.4)$ & $1.83 \pm 0.2$ & $0-27$ & $100 \pm 55$ \\
\hline
\end{tabular}

\section{Discussion}

There have been few studies about the increased iron content in cirrhotic livers, and the definite mechanism by which iron accumulates in cirrhotic liver tissue remains unclear. Most likely, the cause is multifactorial (6). There are some proposed theories, such as increased iron absorption, nutritional deficiencies (especially folate), hypersplenism, hemolysis, and the presence of intra and extrahepatic venous shunts. However, most of these variables resolve after liver transplantation (7).

It is important to know that iron staining can be very high in patients with cirrhosis, and not to interpret every high iron index in cirrhosis as a sign of hemochromatosis (8). The mere presence of granular iron in the liver is referred to as "hemosiderosis", which is most often a morphological observation, rather than a specific disease entity; however, hemochromatosis is a genetic or secondary disease state characterized by the deposition of hemosiderin (8). Liver hemosiderosis in cirrhosis differs, based on the cause of the cirrhosis and, according to the current study, is highest in posthepatitic cirrhosis.

Hepatitis B related cirrhosis and iron overload have rarely been investigated, but it seems that a hepatitis $\mathrm{D}$ co-infection is a risk factor for iron overload (9). There are also reports of brain iron deposition and hepatitis B related iron overload, which can cause neurological problems (10). In contrast to the scarcity of data regarding hepatitis B related cirrhosis and iron overload, there are many reports about the adverse effects of iron overload in patients with chronic hepatitis $C$ related cirrhosis, with regard to treatments and outcomes $(11,12)$.

In our report, there was no significant difference in the iron overload between hepatitis B and C, although the number of hepatitis B cases was far greater than hepatitis C cases (241 vs. 35). There were also 157 cases of cirrhosis with autoimmune (AIH) causes in our study, which showed significant iron overload. However, this association has rarely been reported, and some of the previous reports suggested that the association of an iron overload and $\mathrm{AIH}$ is an indication of hereditary hemochromatosis (13). Our findings showed the presence of this association without any HFE gene mutations.

In 15 cases in the current study, alcoholic cirrhosis was present, and iron overload has been significantly investigated in the alcoholic cause of cirrhosis (14). Previous reports about the whole-body retention of iron have shown a two-fold increase in intestinal iron absorption in chronic alcoholism; however, the underlying mechanism has not yet been identified (15).

In these 1000 cases of cirrhosis, we had 178 patients with pure NASH, but no cirrhosis with the combined etiology of NASH and other causes. After cirrhosis caused by chronic hepatitis, patients with NASH had the highest levels of iron in their liver tissues. Because the iron score contents of the liver was as high as 36 in the patients with $\mathrm{NASH}$, the iron in the liver can be seen everywhere in the Prussian blue stained slides. The mechanisms underlying the iron accumulation in NASH have been shown to be linked with impaired iron export from liver cells, as a consequence of the low expression of the iron export molecules, and the elevated hepcidin concentrations (16). In our study, the least iron content in the liver was found in the patients with biliary cirrhosis, which has also been reported by Batts (8). It seems that stainable iron in biliary cirrhosis is rare and, if present, the amount of stainable iron is low $(17,18)$. In none of our Prussian-blue slides of biliary cirrhosis was the whole tissue loaded with iron. The highest score was 27, and this score was significantly lower than the other types of cirrhosis (i.e. posthepatitic and NASH). Therefore, according to our results, the presence of significant iron deposition in the liver biopsies of the patients with biliary cirrhosis should be considered a clue to the presence of hereditary hemochromatosis.

In this study, the metabolic causes of cirrhosis consisted of a heterogeneous group of unrelated diseases, which cannot be interpreted, because the number of patients suffering from each cause was too low to be interpreted and compared.

\section{Footnotes}

Authors' Contribution:Bita Geramizadeh: designing the protocol, looking at slides, scoring the cases; Yalda Ghazanfari: preparing the slides, analyzing the data, reviewing the charts; Saman Nikeghbalian and Seyed-Ali Malekhosseini: providing the patients.

Funding/Support:This research was based on research protocol No. 2523, supported by the Shiraz University of Medical Sciences.

\section{References}

1. Abu Rajab M, Guerin L, Lee P, Brown KE. Iron overload secondary to cirrhosis: a mimic of hereditary haemochromatosis? Histopathology.2014;65(4):561-9. doi:10.1111/his.12417. [PubMed:24635122]

2. Stuart KA, Fletcher LM, Clouston AD, Lynch SV, Purdie DM, Kerlin P, et al. Increased hepatic iron and cirrhosis: no evidence for an adverse effect on patient outcome following liver transplantation. Hepatology. 2000;32(6):1200-7. doi: 10.1053/jhep.2000.20348. [PubMed: 11093725]

3. Jaskiewicz K, Banach L, Lancaster E. Hepatic siderosis, fibrosis and cirrhosis: the association with hepatocellular carcinoma in high-risk population. Anticancer Res. 1997;17(5B):3897-9. [PubMed: 9427800]

4. Ludwig J, Hashimoto E, Porayko MK, Moyer TP, Baldus WP. Hemosiderosis in cirrhosis: a study of 447 native livers. Gastroenterology. 1997;112(3):882-8. [PubMed: 9041250] 
5. Deugnier Y, Turlin B, le Quilleuc D, Moirand R, Loreal O, Messner $M$, et al. A reappraisal of hepatic siderosis in patients with end-stage cirrhosis: practical implications for the diagnosis of hemochromatosis. Am J Surg Pathol. 1997;21(6):669-75. [PubMed: 9199645]

6. Parolin MB, Batts KP, Wiesner RH, Bernstein PS, Zinsmeister AR, Harmsen WS, et al. Liver allograft iron accumulation in patients with and without pretransplantation hepatic hemosiderosis. Liver Transpl. 2002;8(4):331-9. doi:10.1053/jlts.2002.30980. [PubMed: 11965576]

7. Cotler SJ, Bronner MP, Press RD, Carlson TH, Perkins JD, Emond MJ, et al. End-stage liver disease without hemochromatosis associated with elevated hepatic iron index. J Hepatol.1998;29(2):25762. [PubMed: 9722207]

8. Batts KP. Iron overload syndromes and the liver. Mod Pathol 2007;20 Suppl 1:S31-9. doi: 10.1038/modpathol.3800715. [PubMed: 17486050]

9. Sebastiani G, Tempesta D, Alberti A. Hepatic iron overload is common in chronic hepatitis B and is more severe in patients coinfected with hepatitis D virus. J Viral Hepat. 2012;19(2):e170-6. doi: 10.1111/j.1365-2893.2011.01508.x. [PubMed: 22239515]

10. Lin D, Ding J, Liu JY, He YF, Dai Z, Chen CZ, et al. Decreased serum hepcidin concentration correlates with brain iron deposition in patients with HBV-related cirrhosis. PLoS One. 2013;8(6):e65551. doi:10.1371/journal.pone.0065551. [PubMed:23776499]

11. Lin TJ, Liao LY, Lin SY, Lin CL, Chang TA. Influence of iron on the severity of hepatic fibrosis in patients with chronic hepatitis $C$. World J Gastroenterol. 2006;12(30):4897-901. [PubMed: 16937477]
12. Lambrecht RW, Sterling RK, Naishadham D, Stoddard AM, Rogers T, Morishima C, et al. Iron levels in hepatocytes and porta tract cells predict progression and outcomes of patients with advanced chronic hepatitis C. Gastroenterology. 2011;140(5):1490500 e3. doi:10.1053/j.gastro.2011.01.053. [PubMed: 21335007]

13. An IC, Tiwari AK, Ameda S, Laird-Fick HS. Autoimmune hepatitis: diagnostic dilemma in the setting of suspected iron overload. Case Rep Gastrointest Med. 2013;2013:872987. doi: 10.1155/2013/872987. [PubMed: 24024049]

14. Dostalikova-Cimburova M, Kratka K, Stransky J, Putova I, Cieslarova B, Horak J. Iron overload and HFE gene mutations in Czech patients with chronic liver diseases. Dis Markers. 2012;32(1):65-72. doi:10.3233/DMA-2012-0861. [PubMed:22297603]

15. Iqbal T, Diab A, Ward DG, Brookes MJ, Tselepis C, Murray J, et al. Is iron overload in alcohol-related cirrhosis mediated by hepcidin? World J Gastroenterol. 2009;15(46):5864-6. [PubMed:19998511]

16. Aigner E, Weiss G, Datz C. Dysregulation of iron and copper homeostasis in nonalcoholic fatty liver. World J Hepatol. 2015;7(2):177-88. doi: 10.4254/wjh.v7.i2.177. [PubMed: 25729473]

17. Huang YH, Yu-Hsieh H, Huang CC, Shin-Mu VT, Tai MH, Chen $\mathrm{CL}$, et al. Liver hepcidin and stainable iron expression in biliary atresia. Pediatr Res. 2006;59(5):662-6. doi: 10.1203/01. pdr.0000214974.55049.f1.[PubMed:16627878]

18. Jain S, Agarwal S, Tamhankar P, Verma P, Choudhuri G. Lack of association of primary iron overload and common HFE gene mutations with liver cirrhosis in adult Indian population. Indian J Gastroenterol. 2011;30(4):161-5. doi: 10.1007/s12664-011-0109-5. [PubMed: 21822737] 